\title{
Waiting times for prostate cancer diagnosis in KwaZulu-Natal, South Africa
}

\author{
K Singh, ${ }^{1}$ MB ChB; E H Abdel Goad, ${ }^{1}$ MB ChB, FCUrol, MMedSc, FEBU, FRCS (Ire); S S Ramklass, ${ }^{2}$ BPhysiother, MEd, DEd \\ ${ }^{1}$ Department of Urology, School of Clinical Medicine, College of Health Sciences, Nelson R Mandela School of Medicine, \\ University of KwaZulu-Natal, Durban, South Africa \\ ${ }^{2}$ College of Health Sciences, Nelson R Mandela School of Medicine, University of KwaZulu-Natal, Durban, South Africa
}

\author{
Corresponding author: KSingh (sngkir@gmail.com)
}

\begin{abstract}
Background. There is currently no evidence in the South African (SA) literature to suggest how long patients with clinically suspected prostate cancer (an elevated prostate-specific antigen level or abnormal findings on digital rectal examination) wait to have a prostate biopsy.

Objectives. To improve the overall efficiency of the prostate biopsy service offered at St Aidan's Regional Hospital, Durban, SA, by quantifying the burden of disease and waiting times and to identify potential delays in management outcomes, thereby helping to alleviate patient anxiety during the stressful period of investigation.

Methods. We did a retrospective folder review of patients who underwent trans-rectal prostate biopsy at St Aidan's Hospital, where the vast majority of prostate biopsies in the KwaZulu-Natal state healthcare sector are performed, from January to June 2013. The Statistical Package for Social Sciences was used for data analysis.

Results. One hundred and six patients (mean age 67.6 years, 69.8\% black Africans) underwent biopsy during the 6-month study period; $49.1 \%$ were found to have adenocarcinoma, and of the $80.1 \%$ of these who had a bone scan, $73.8 \%$ had skeletal metastases $(p=0.1379)$. The median period of time from referral to biopsy was 55 days, from referral to first follow-up date (when the diagnosis is given and treatment options discussed or instituted) 100 days, and from biopsy to first follow-up date (i.e. waiting period to retrieve histological diagnosis) 36 days.

Conclusion. Despite the late presentation of prostate cancer in KZN, patients are waiting an average of 3 months from initial referral for a prostate biopsy to institution of definitive management.
\end{abstract}

S Afr Med J 2015;105(6):484-486. DOI:10.7196/SAMJ.9192

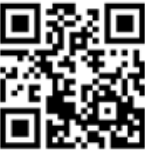

Prostate cancer is a very common malignancy in many countries. In the USA it accounts for almost a third $(31.2 \%)$ of all cancers, ranking second only to lung cancer as the cause of death in men (11.7\%). ${ }^{[1]}$ There seems to be a paucity of evidence regarding the epidemiology of prostate cancer in sub-Saharan Africa. ${ }^{[2-3]}$ It is, however, known that men of African descent appear to suffer disproportionately more from prostate cancer compared with men of other races or ethnicities, ${ }^{[4]}$ and present with more advanced disease. ${ }^{[5-8]}$

To date most, if not all, of the epidemiological studies on prostate cancer in South Africa (SA) have been undertaken by Heyns and colleagues in the Western Cape Province, ${ }^{[5,9-13]}$ and their findings have influenced national guidelines and policy. However, it remains to be established whether these findings are consistent among the various provinces of SA, in part because of different racial composition and socioeconomic status of populations.

With the imminent roll-out of National Health Insurance (NHI) in SA, there is a need to quantify the burden of prostate cancer on the health service. In a resource-deprived setting such as KwaZulu-Natal Province (KZN), human resources and infrastructure need to be allocated appropriately to enhance the quality of care that patients receive. ${ }^{[14]}$ There is currently no literature in SA regarding how long patients with suspected prostate cancer wait to have a prostate biopsy in the public healthcare sector, and how long they wait to initiate treatment.

\section{Objectives}

To form a framework to improve the overall efficiency of the prostate biopsy service at St Aidan's Hospital, Durban, which serves as a specialist urology referral centre. We described the patient profile, referral pattern and pathological burden of disease, calculated the waiting period in days from peripheral hospital referral to biopsy date, and attempted to identify high-risk groups in which referral should be expedited and to identify potential delays in management.

\section{Methods}

A retrospective folder review of patients who had undergone transrectal prostate biopsy for clinically suspected prostate cancer was undertaken at the Urology Cinic at St Aidan's Hospital from January 2013 to June 2013. Patients were referred to the service directly from peripheral health facilities and local hospitals in the eThekwini district, KZN.

All patients had sextant trans-rectal ultrasound-guided (TRUS) biopsies for the first time, and only those with confirmed histological findings were included.

The study was three-tiered and aimed at defining the scope of the burden of disease on the service, calculating the waiting times and describing the referral patterns. Patients self-assigned their race as black African, white, Indian or coloured (of mixed ancestry).

Time intervals were calculated from dates obtained from referral notes. Three different time intervals were calculated: (i) from referral to biopsy, i.e. the number of days a patient waited to receive a biopsy after being referred to the service; (ii) from referral to first followup, i.e. the number of days a patient waited to receive a histological diagnosis, and for treatment options to be discussed or instituted; and (iii) from biopsy date to first follow-up date, i.e. waiting period to retrieve a histological diagnosis, or histology processing time. 
Because of incomplete and manual record keeping, we were unable to do accurate clinical staging according to the 2002 TNM classification. However, patients were risk-stratified according to the D'Amico risk stratification, ${ }^{[15]}$ using prostate-specific antigen (PSA) in most instances. Haematogenous metastases were detected by skeletal scintigraphy (bone scan). Indications for prostate biopsy included a serum PSA level $>4 \mathrm{ng} / \mathrm{mL}$ or suspicious findings on digital rectal examination (DRE).

\section{Statistical analysis}

Data were captured using Microsoft Excel and analysed using the Statistical Package for Social Sciences (SPSS version 21) in consultation with a biostatistician. Comparison of means was performed using Student's $t$-test for parametric data and the Mann-Whitney and KruskalWallis tests for non-parametric data. Fisher's exact test was used for contingency table analysis. A two-tailed $p$-value of $<0.05$ was accepted as statistically significant.

\section{Ethical approval}

Ethical approval for the study was granted by the University of KwaZulu-Natal Biomedical Research Ethics Committee and the KwaZulu-Natal Provincial Health and Research Ethics Committee.

\section{Results}

One hundred and six patients (mean age 67.6 years (standard deviation 7.51)) underwent TRUS biopsy. Of these patients, $69.8 \%$ $(n=74)$ were classified as black, $4.7 \%(n=5)$ as white, $13.2 \%(n=14)$ as Indian and $12.3 \%(n=13)$ as coloured.

Almost half of the patients $(49.1 \%, n=52)$ were found to have adenocarcinoma. Other findings included benign prostatic hyperplasia, prostatitis, and inadequate specimen for analysis. The distribution of histological findings is illustrated in Fig. 1. The majority of malignancies (75.0\%, $n=39$ ) were found in black patients (Fig. 2).

According to the D'Amico risk stratification, $68.9 \%(n=51)$ of black patients were classified as high risk v. $4.7 \%$ of whites $(n=5), 13.2 \%$ of Indians $(n=14)$ and $12.3 \%$ of coloureds $(n=13)$, which was statistically significant $(p<0.001)$. The median PSA level (Fig. 3) of black African patients with prostate cancer was $42.2 \mathrm{ng} / \mathrm{mL}$ v. $12.8 \mathrm{ng} / \mathrm{mL}$ for whites, $10.3 \mathrm{ng} / \mathrm{mL}$ for Indians and $14.2 \mathrm{ng} / \mathrm{mL}$ for coloureds, which was also statistically significant $(p<0.001)$. The median PSA level of patients with confirmed carcinoma was $133.5 \mathrm{ng} / \mathrm{mL}$, as opposed to $16.0 \mathrm{ng} / \mathrm{mL}$ for those without cancer $(p<0.01)$. A relationship was observed between initial PSA level and Gleason score $(p=0.01)$. We were unable to stage patients according to the TNM classification, as in $36.8 \%$ of folders the DRE findings were not adequately documented. Of the 52 patients with histologically confirmed adenocarcinoma, $80.1 \%(n=42)$ received a bone scan; in $73.8 \%(n=31)$ skeletal scintigraphy confirmed metastases, $8(18.6 \%)$ had no metastases, and $4(9.3 \%)$ had equivocal findings ( $p=0.1379)$.

With regard to treatment modalities, $92.5 \%$ of patients $(n=98)$ received some form of medication; only $8.5 \%(n=9)$ underwent surgical intervention, while $4.7 \% \quad(n=5)$ received external-beam radiation. Medication included alpha-blockers $(n=32)$, antibiotics $(n=4)$, androgen-deprivation therapy (ADT) $(n=47)$ and combination regimens $(n=62)$. Of the patients with confirmed adenocarcinoma, $90.4 \%$ received ADT.

The majority of referrals $(59.4 \%, n=63)$ were from the King Edward VIII Hospital (KEH) complex, 22.6\% were from Addington Hospital (AH), 16.0\% were from R K Khan Hospital (RKKH), and only $1.9 \%$ were from private practitioners. Of those with confirmed prostate adenocarcinoma $(n=52), 55.8 \%(n=29)$ were referred from $\mathrm{KEH}, 25.0 \%(n=13)$ from $\mathrm{AH}, 17.3 \%(n=9)$ from RKKH and $1.9 \%$ $(n=1)$ from private practice.

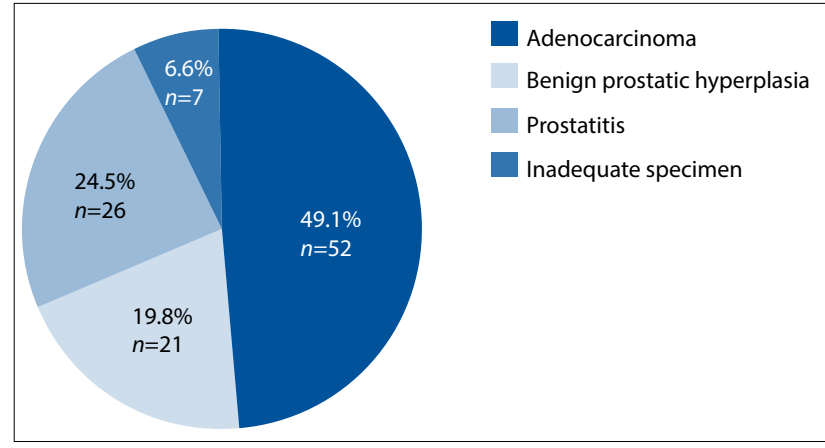

Fig. 1. Categorisation of histological outcomes.

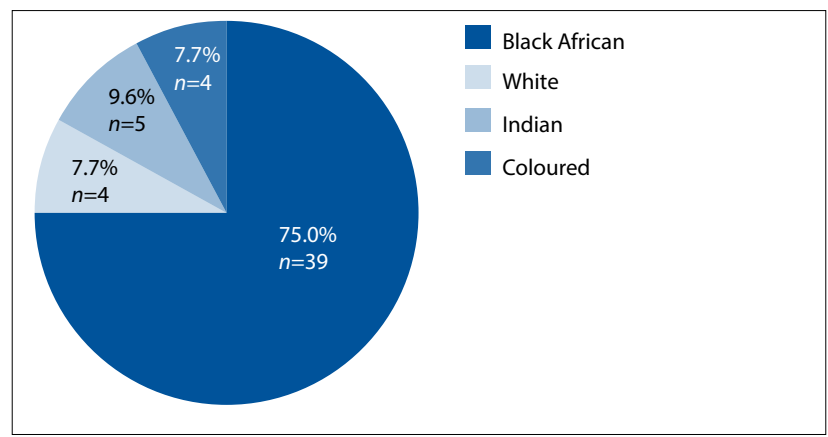

Fig. 2. Racial distribution of prostate cancer.

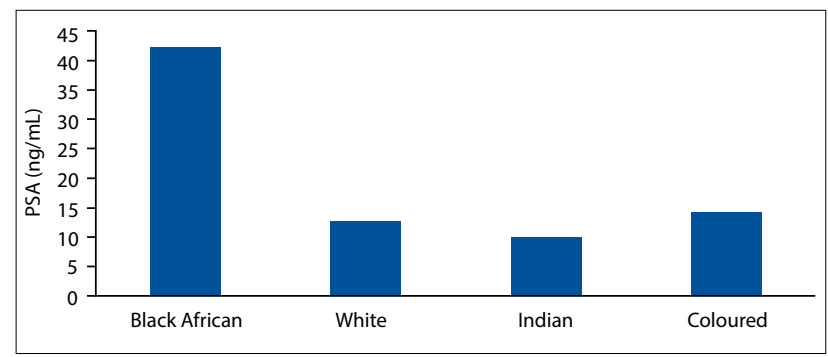

Fig. 3. Median PSA levels in the different races with prostate cancer.

The median period of time from referral to biopsy date was 55 days, from referral to first follow-up date 100 days, and from biopsy date to first follow-up date 36 days (Fig. 4). No relationship was observed between referral centre and any of the waiting periods ( $p>0.05)$.

\section{Discussion}

The patients in this study were more representative of the demographics of the general SA population than the larger 10-year retrospective review of 901 patients with prostate cancer managed by the urological oncology clinic at Tygerberg Hospital, Western Cape, by Heyns et al. ${ }^{[5,16]}$ The Western Cape study ${ }^{[5]}$ demonstrated a predominance $(59.8 \%)$ of white patients with prostate cancer, black patients accounting for only $32.3 \%$. This differs from the findings of our study, in which there was a predominance of blacks $(69.8 \%, n=74)$, and whites accounted for only $13.2 \%(n=14)$. The median PSA level of our black patients with prostate cancer was $42.2 \mathrm{ng} / \mathrm{mL}(n=74)$ and that for whites was $12.8 \mathrm{ng} / \mathrm{mL}(n=5)$, in comparison with findings in the Western Cape ${ }^{[5]}$ of $105 \mathrm{ng} / \mathrm{mL}$ and $19.6 \mathrm{ng} / \mathrm{mL}$, respectively.

Owing to the lack of understanding of the disease profile in KZN, and lack of an updated National Cancer Registry, it is very difficult to draw guidelines, allocate resources and streamline biopsy services with the aim of establishing an efficient service and identifying curable disease. Patients are waiting for an average of 3 months (100 days) from the initial referral date to presumed diagnosis and definitive 
Table 1. Reasons for delay in diagnosis

Patient factors
- Lack of personal health awareness
- Only seeking medical intervention
when symptomatic or traditional
medicine has failed

Healthcare provider factors Infrastructure factors

- Inadequate gatekeeper knowledge of DRE, - Lack of dedicated prostate biopsy clinics PSA screening and when to appropriately - Lack of equipment - ultrasound machines with refer rectal probes and biopsy needles

- Belief that prostate cancer is a slowly - Overbooked clinics progressive disease that does not need to be - Lengthy pathology processing time referred timeously

- Inadequate human resource training and allocation
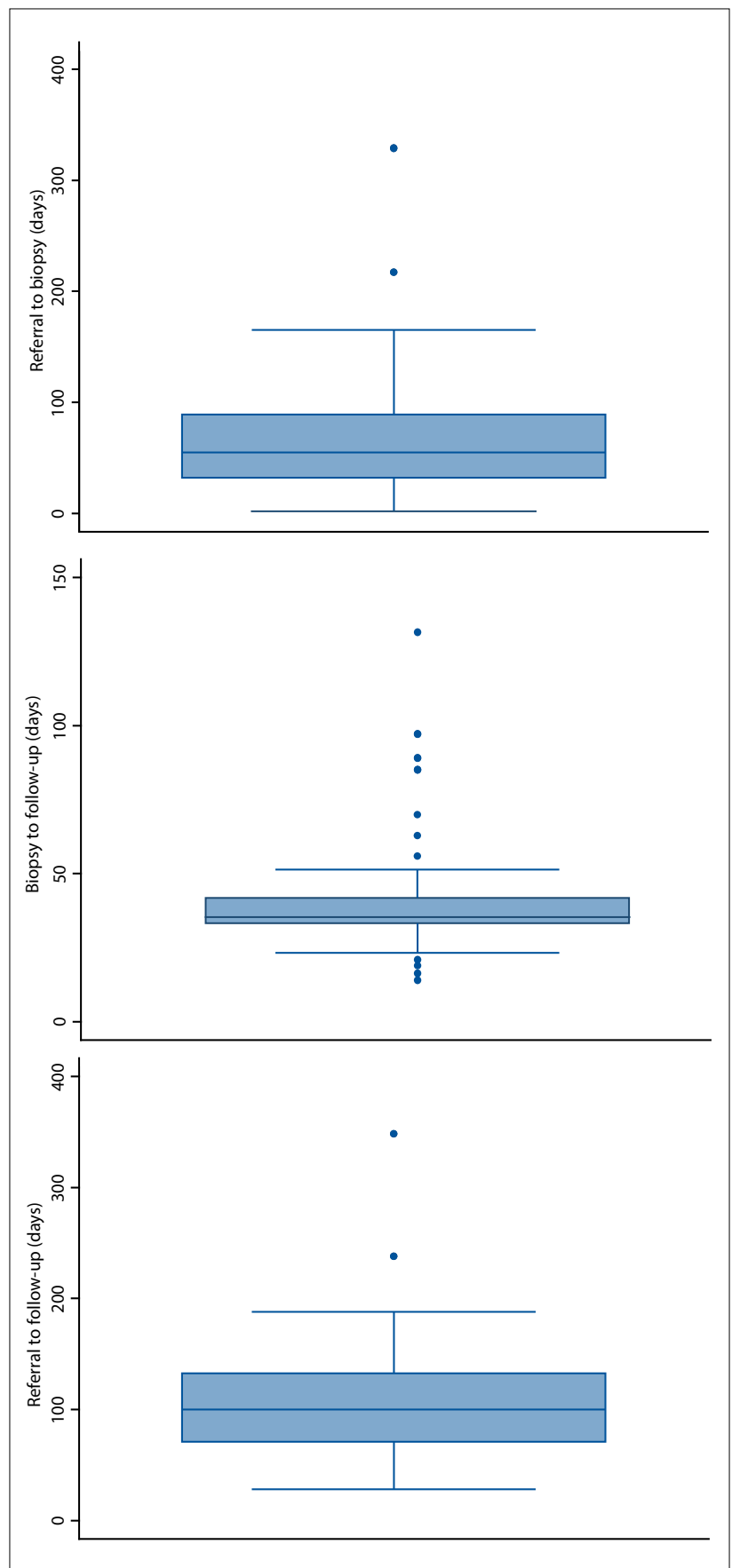

Fig. 4. Waiting times for the different time intervals observed.

management, with only $7.7 \%(n=4)$ with prostate cancer qualifying for a potentially curative procedure. It is difficult to comment on this figure further, as lack of adequate record keeping was a major

challenge and patients could not be staged adequately. However, almost two-thirds $(n=31)$ of patients with confirmed adenocarcinoma were found to have had advanced disease at presentation. A future larger prospective study should be performed in KZN, similar to that of Heyns et al. ${ }^{[9]}$ who found that potentially curative treatment could be offered to $37.5 \%$ of patients with clinically localised prostate cancer.

Postulated reasons for the delay in diagnosis include but are not limited to patient, healthcare provider and infrastructure factors (Table 1).

\section{Conclusion}

Before this study, we were uncertain of the scope of pathology encountered at the St Aidan's Hospital complex. We found that almost half of all patients referred to our service have prostate cancer, and that almost two-thirds of those with histological confirmation of prostate cancer will have metastatic disease at presentation. As shown in other larger studies ${ }^{[5]}$ the vast majority of black patients with prostate cancer present with high PSA levels, indicating advanced disease in most cases. Patients are waiting an average of 3 months to receive a diagnosis and plan of treatment after being referred.

As the number of patients increases with the arrival of NHI, waiting times for treatment are likely to continue to increase, potentially resulting in additional treatment delays.

\section{References}

1. Max W, Rice DP, Sung HY, et al. The economic burden of prostate cancer, California, 1998. Cancer 2002;94(11):2906-2913. [http://dx.doi.org/10.1002/cncr.10532]

2. Rebbeck TR, Devesa SS, Chang BL, et al. Global patterns of prostate cancer incidence, aggressiveness, and mortality in men of African descent. Prostate Cancer 2013 (2013), Article ID 560857. [http:// dx.doi.org/10.1155/2013/560857]

3. Boyle P, Levin B. World Cancer Report 2008. Lyon: IARC Press, 2008

4. Odedina FT, Akinremi TO, Chinegwundoh F, et al. Prostate cancer disparities in black men of African descent: A comparative literature review of prostate cancer burden among black men in the United States, Caribbean, United Kingdom, and West Africa. Infect Agent Cancer 2009;4(Suppl 1):S2. [http:// dx.doi.org/10.1186/1750-9378-4-S1-S2

5. Heyns CF, Fisher M, Lecuona A, van der Merwe A. Prostate cancer among different racial groups in the Western Cape: Presenting features and management. S Afr Med J 2011;101(4):267-270.

6. Evans HS, Moller H. Recent trends in prostate cancer incidence and mortality in southeast England. Eur Urol 2003;43(4):337-341. [http://dx.doi.org/10.1016/S0302-2838(03)00085-X]

7. Sandblom G, Varenhorst E, Lofman O, et al. Clinical consequences of screening for prostate cancer: 15 years follow-up of a randomised controlled trial in Sweden. Eur Urol 2004;46(7):717-723. [http:// dx doi.org/10.1016/j. eururo.2004.08.011]

8. Paquette EL, Sun L, Paquette LR, et al. Improved prostate cancer-specific survival and other disease parameters: Impact of prostate-specific antigen testing. Urology 2002;60(5):756-759. [http://dx.doi. org/10.1016/S0090-4295(02)01960-X]

9. Heyns CF, Naude AM, Visser AJ, et al. Early diagnosis of prostate cancer in the Western Cape. S Afr Med J 2001;91(8):679-684.

10. Heyns CF, Mathee S, Isaacs A, Kharwa A, de Beer PM, Pretorius MA. Problems with prostate specific antigen screening for prostate cancer in the primary healthcare setting in South Africa. BJU Int 2003;91(9):785-788. [http://dx.doi.org/10.1046/j.1464-410X.2003.04241.x ]

1. Heyns CF, Naude AM, Ahmed G, Stopforth HB, Stellmacher GA. Serum prostate-specific antigen as a surrogate for the histological diagnosis of prostate cancer. S Afr Med J 2001;91(8):685-689.

12. Heyns CF, van der Merwe A. Prostate specific antigen - brief update on its clinical use. S Afr Fam Pract 2008;50(2):19-24.

13. Heyns $\mathrm{CF}$, van der Merwe A. Prostate cancer management - helping your patient choose what is best for him. S Afr Fam Pract 2008;50(5):27-34.

14. Heyns CF. Urology in South Africa. J Maroc Urol 2006;2:4-6. http://lazraqinfo.com/JMU/PDFn2/ Heynssouthafrica.pdf (accessed 8 May 2015).

15. D’Amico AV, Chen MH, Malkowicz SB, et al. Lower prostate specific antigen outcome than expected following radical prostatectomy in patients with high grade prostate cancer and a prostatic specific
follicis antigen level of $4 \mathrm{ng} / \mathrm{ml}$ or less. J Urol 2002;167(5):2025-2030; discussion 2030-2031. [http://dx.doi. org/10.1016/S0022-5347(05)65076-8]

16. Census 2011: Census in Brief. Pretoria: Statistics South Africa, 2012.

Accepted 20 January 2015. 